\title{
IL6 VALUES FROM THE BLOOD OF UMBILICAL CORD IN PREDICTION OF PERINATAL BRAIN DAMAGE
}

\author{
F. Skokic \\ Department of Neonatology, Clinic for Gynecology and Obstetrics, University Clinical Center, Tuzla, \\ Bosnia-Herzegovina
}

Aim: To examine predictive value of interleukin 6 (IL-6) in the blood of umbilical cord in detection of early brain damage, compering to ultrasonic exam of the brain.

Methods: Prospective study it included 315 newborns with the body weight from 500 to 2000 grams. IL-6 values were analyzed from the blood of umbilical cord. Perinatal brain damages were following by ultrasound exam of the brain and it was repeated first and third day of life.

Results: $117 / 315$ (37, 1\%) newborns had brain damage. 61/117 (52, 1\%) newborns had HIC, 38/117 (32, 5\%) newborns had HIE, and 18/117 (15, 4\%) newborns had HIC and HIE, all confirmed by ultrasound exam. IL6 values in all three groups were increased, but not significant $(p>0.05)$. The newborns with HIC had median IL6 16,2 pg/ml, slightly higher median IL6 $(21,1 \mathrm{pg} / \mathrm{ml})$ had newborns with HIE, and third group of newborns (HIE and HIC) had median IL6 $19,5 \mathrm{pg} / \mathrm{ml}$. The specificity of IL6 compared to ultrasound was $77 \%$ for HIC, $71 \%$ for HIE and $67 \%$ for both HIC and HIE. The sensitivity of IL6 was $34 \%$ for HIC, $25 \%$ for HIE and very low for HIC and HIE 13\%, all compared to ultrasound.

Conclusion: It could be used for selection of newborns that need continuous following and treatment in early neonatal period. 